\title{
LINEAR FUNCTIONAL TRANSFORMATIONS IN GENERAL SPACES†
}

\author{
BY T. H. HILDEBRANDT
}

1. Introduction. An abstract theory of linear functional transformations has as guide linear transformations in a finite or denumerably infinite set of variables, linear integral transformations and equations associated with these. The desire to proceed symbolically and replace details by general procedure seems to be inherent in the situation. Pincherleł is perhaps one of the first great exponents, so that he even seems to have anticipated some of the famous results of integral equations by a number of years. E. H. Moore§ set himself the task of unifying the Fredholm theory of integral equations and algebraic equations in finitely and infinitely many variables, and has succeeded in setting up a system which indicates in a host of special cases a valid and elegant method of procedure analogous to the Fredholm integral equation theory. Volterra $\uparrow$ has devised an elegant theory of linear integral and associated operations based on the notion of permutability or commutativity of operations.

The theory to which the main portion of this address is devoted has not been, in the main, presented as such in published form. It is, however, obvious that F. Riesz in his book entitled Les Equations Linéaires à une Infinité d'Inconnus $\|$ and his paper, Lineare Funktionalgleichungen $\dagger \dagger$ has had in mind the generalizations treated here. As a consequence, what is given here is in the main hardly new, excepting that by presenting it from the point of view of a general basis, there is a gain in elegance and simplicity for the non-pathological results.

$\dagger$ An address delivered at the Summer Meeting of the Society at Providence, September 10,1930, by invitation of the program committee.

$\ddagger$ See Notice sur les travaux, Acta Mathematica, vol. 46 (1925), pp. 341-362, especially p. 347, and pp. 351-354; L'Operazioni Distributive, Bologna, 1901; Encyclopédie des Sciences Mathématiques, vol. II, 26 (1912).

§ See this Bulletin, vol. 28 (1912), pp. 334-362; Proceedings of the Cambridge International Congress, 1912, vol. I, pp. 230-255. etc.

T See for instance Legons sur les Fonctions des Lignes, Paris, 1913, Chap. 9,

|| Paris, 1913; this book will be cited in what follows as Riesz, Inf. Inc.

†† Acta Mathematica, vol. 41 (1917), pp. 75-88; this paper will be cited in what follows as Riesz, $L F G$. 
The first five sections of this paper are devoted to a presentation of the theory of the linear limited transformation, indicating results which can be derived in a complete vector space. The last sections give a brief account of the generalization of the Hilbert theory of symmetric kernels, and infinite limited matrices, on a more special type of linear vector space. A general basis in which the fundamental form is obtained constructively was derived by $\mathrm{E}$. H. Moore in his second theory $\dagger$ which aimed at a generalization of the Hilbert-Hellinger theory. The postulational basis used here was given by von Neumann.t

2. The Space. We shall assume as basis a class of elements $\xi$ constituting a linear vector space $₫$, that is, a space satisfying the following conditions:

(A) There is defined addition, commutative and associative with respect to the elements; and multiplication by complex numbers, commutative, associative, and distributive; further a unique zero element 0 , identical with $0 \cdot \xi$ for every $\xi$ of the space.

(B) There exists a norm or metric transforming each element into a positive real number, satisfying the conditions

(1) $\left\|\xi_{1}+\xi_{2}\right\| \leqq\left\|\xi_{1}\right\|+\left\|\xi_{2}\right\|$;

(2) $\|a \xi\|=|a| \cdot\|\xi\|$, for every complex number $a$;

(3) $\|\xi\|=0$, if and only if $\xi=0$.

(C) The space is complete, that is, if the sequence $\xi_{n}$ satisfies the condition $\lim _{n, m}\left\|\xi_{n}-\xi_{m}\right\|=0$, then there exists a $\xi$ such that $\lim _{n}\left\|\xi_{n}-\xi\right\|=0$. If the space $\subseteq$ is not complete, it is obvious that completeness is extensionally attainable after the manner of deriving the real number system from the rational number system.

As examples of spaces satisfying the above postulates, we might note the following: $\mathbb{1}$

$\dagger$ Available at present only in lecture notes, excepting some indications in Mathematische Annalen, vol. 86 (1922), pp. 34, etc.

$\ddagger$ Göttinger Nachrichten, 1927.

$\S$ See Banach, Fundamenta Mathematicae, vol. 3 (1922), p. 135; Wiener, Bulletin de la Société Mathématique de France, vol. 49 (1921), pp. 123-124; Fréchet, Les Espaces Abstraits, pp. 125-126.

I This list is intended to be illustrative, not exhaustive. Hahn, Monatshefte, vol. 32 (1922), pp. 1-88, gives a large number of illustrations. 
Group I. Let $\xi$ be a function on the set of all positive integers, that is, a sequence $\left\{x_{p}\right\},(p=1,2,3, \cdots)$.

I.1 $1_{e}$. The space $\subseteq$ consists of all sequences for which $\sum\left|x_{p}\right|^{1+\bullet}$ converges, the norm $\|\xi\|$ being the $(1+e)$ th root of this sum. For $e=1$, we get Hilbert space.

I.2. The space $\subseteq$ consists of all convergent series, with $\|\xi\|$ $=L U B\left|\sum_{1}^{n} x_{p}\right|$, where $L U B$ is the least upper bound.

I.3. The space $\subseteq$ consists of all sequences having a limit, with $\|\xi\|=L U B\left|x_{p}\right|$.

I.3. The space $\subseteq$ consists of all sequences having zero as limit, with same norm as in I.3. Obviously there exists a one to one correspondence between I.2, 3, and $3_{0}$.

I.4. The space $\subseteq$ consists of all bounded sequences with norm $\|\xi\|=L U B\left|x_{p}\right|$.

Group II. Let $\xi$ be a real or complex valued function $x(p)$ on the linear interval $0 \leqq p \leqq 1$.

II. $1_{e}$. The space $\subseteq$ consists of all measurable functions for which the Lebesgue integral $\int_{0}^{1}|x(p)|{ }^{1+e} d p$ exists, with $\|\xi\|$ the $(1+e)$ th root of this integral.

II.2. The space $\mathfrak{S}$ consists of all measurable functions, bounded except for a set of zero measure, $\|\xi\|$ being the effective upper bound of $|x(p)|$, that is, the greatest lower bound of the numbers $M$ such that the set of elements $p$ for which $|x(p)|>M$ is of measure zero.

II.3. The space $\subseteq$ consists of all continuous functions, $\|\xi\|$ being the maximum of $|x(p)|$.

II.4. The space $\subseteq$ consists of all functions of bounded variation, $\|\xi\|$ being the total variation on the interval.

For two interesting linear spaces, it is not known that they are of this vector type; these are the set of all sequences, with $\lim \xi_{n}=\xi$ defined as $\lim x_{n}(p)=x(p)$ for every $p$, and the set of all measurable functions with limit defined as convergence in the measure, that is, for every $e$, the measure of the set of elements $p$ for which $\left|x_{n}(p)-x(p)\right|>e$ approaches zero with $n$. In each of these cases there exists a metric $\dagger$ giving an equivalent definition of limit but the metric does not satisfy the multiplicative condition (2).

† See, for instance, Fréchet, Rendiconti di Palermo, vol. 22 (1906), p. 38; and Bulletin of Calcutta Mathematical Society, vol. 11 (1921), pp. 190, etc. 
As is customary, we shall call a subset $\mathfrak{S}_{0}$ of the space $\mathfrak{S}$ linear if every linear combination of elements of $\mathfrak{S}_{0}$ belongs to $\mathfrak{\Im}_{0}$; closed if the limit of a sequence of elements of $\mathfrak{\Im}_{0}$ also belongs to $\mathfrak{\Im}_{0}$. Of importance are the linear and closed extensions of a subset $\mathfrak{\Im}_{0}$, attainable by adding to $\mathfrak{\subseteq}$ all linear combinations of its elements, that is, the linear extension of $\mathfrak{S}_{0}$, and then the limits of all convergent sequences of this latter class. If $\varsigma_{0}$ is the linear closed extension of a finite number of elements of $\mathfrak{S}$, it is said to be of finite dimension. An important theorem in this connection is due to Riesz $\dagger$ that a necessary and sufficient condition that a linear closed subset $\mathfrak{\Im}_{0}$ of $\mathfrak{\subseteq}$ be of finite dimension is that every bounded set of elements be compact (that is, contain a subsequence having a limit).

Next to the sets of finite dimension are the sets or spaces of denumerably infinite dimension, that is, in which there exists a sequence of elements $\xi_{1}, \xi_{2}, \cdots, \xi_{n}, \cdots$ whose linear closed extension is the space. The spaces I. $1_{e}, \mathrm{I} .2, \mathrm{I} .3, \mathrm{II} .1_{e}, \mathrm{II} .3$ have this property, but not the spaces I.4, II.2 and II.4.

A set $(\$)$ is called a fundamental set, if $\mathbb{S}$ is the linear closed extension of $(5)$.

3. Linear Limited Operations. A linear limited operation $L(\xi)$ on $\mathfrak{S}$ satisfies the following three conditions:

(a) To every $\xi$ of $\subseteq$ there corresponds a real or complex number.

(b) If $\xi_{1}$ and $\xi_{2}$ are elements of $\subseteq$ and $a_{1}$ and $a_{2}$ complex numbers, then

$$
L\left(a_{1} \xi_{1}+a_{2} \xi_{2}\right)=a_{1} L\left(\xi_{1}\right)+a_{2} L\left(\xi_{2}\right) .
$$

(c) There exists a number $M$ such that for every $\xi$ of $\mathfrak{S}$, we have $|L(\xi)| \leqq M\|\xi\|$. The greatest lower bound of all possible $M$ 's satisfying this condition will be called the modulus $M_{L}$ of $L$. The condition (c) is equivalent in our space $\subseteq$ to continuity, that is, if $\lim _{n} \xi_{n}=\xi$, then $\lim _{n} L\left(\xi_{n}\right)=L(\xi)$, continuity at one point being sufficient. $\$$ In the case of metric spaces not satisfying the multiplicative condition (2) on the norm, this equivalence is not possible, and it seems preferable to discuss linear continuous operations.

The question, what is the most general linear limited operation on a given space $\mathfrak{S}$, is of interest, but has not yet received

$\dagger$ Riesz, $L F G$, pp. 77-79.

$\ddagger$ See Banach, loc. cit., p. 152. 
exhaustive treatment. The following results are comparatively well known: $\dagger$

I.1 $1_{e}$, with $e>0 . L(\xi)=\sum a_{p} x_{p}$, where $\sum\left|a_{p}\right|^{1+1 / e}<\infty$, that is, $a_{p}$ belongs to $\mathrm{I} \cdot 1_{1 / e}$.

I. $1_{0}$, that is, $e=0 . L(\xi)=\sum a_{p} x_{p}$, where the set $a_{p}$ is bounded, that is, $a$ belongs to space I.4.

I.2 $L(\xi)=\sum a_{p} x_{p}$, with $\sum\left|a_{p}-a_{p+1}\right|<\infty$.

I.3 $L(\xi)=\sum a_{p} x_{p}$, with $\sum\left|a_{p}\right|<\infty$, that is, $a_{p}$ is of I.1.

I.3 $L(\xi)=\sum a_{p} x_{p}+a_{0} x_{0}$, where $\lim _{p} x_{p}=x_{0}$ and $\sum\left|a_{p}\right|<\infty$.

II.1 $(e>0$. $) \quad L(\xi)=\int \alpha(p) x(p) d p$, where $\int|\alpha(p)| 1+1 / e$ exists.

II.1 ${ }_{0}(e=0$.) Same form, but $\alpha$ is bounded except for a set of zero measure, that is, of II.2.

II.3 $L(\xi)=\int x(p) d \alpha(p), \alpha$ of bounded variation.

The most general linear limited operations in the spaces I.4, II. 2 and II.4 do not seem to have been determined, the problem in the cases listed being simplified because of the separability of the space. Examples of linear operations in II.4 are $\int \alpha(p) d x(p)$, where $\alpha(p)$ is continuous, or more generally if a LebesgueStieltjes integral, then $\alpha(p)$ may be a Borel measurable function. On the other hand $\sum_{i}\left[x\left(p_{i}+0\right)-x\left(p_{i}-0\right)\right]$, where $p_{i}$ are the points of discontinuity of $x$ and the summation is extended over these, is obviously linear limited, but not expressible in integral form.

The following theorem is interesting in the theory of linear operations.

THEOREM 1. If $L_{n}(\xi)$ is a sequence of linear limited operations converging to the operation $L(\xi)$, then the sequence is uniformly limited (that is, the sequence of moduli $M_{L_{n}}$ is bounded) and $L(\xi)$ is a linear limited operation. $\$$

It is possible to generalize this theorem in a number of ways. First the linearity condition on $L_{n}$ may be replaced by a property similar to the triangle property of the norm, namely,

† See Riesz, Mathematische Annalen, vol. 69 (1910), p. 475; Annales de l'École Normale, (3), vol. 31 (1914), pp. 9-14; Helly, Monatshefte, vol. 31 (1921), p. 84; Hahn, loc. cit.

$\ddagger$ See Banach, loc. cit., p. 157. 


$$
\left|L\left(a_{1} \xi_{1}+a_{2} \xi_{2}\right)\right| \leqq\left|a_{1}\right|\left|L\left(\xi_{1}\right)\right|+\left|a_{2}\right|\left|L\left(\xi_{2}\right)\right|
$$

for all $\xi_{1}$ and $\xi_{2}$ of $\subseteq$ and all complex numbers $a_{1}$ and $a_{2}$. Then the condition that the sequence of values $L_{n}(\xi)$ converge for every $\xi$ may be replaced by assuming that for each $\xi$ the sequence of numbers $L_{n}(\xi)$ is bounded. Finally the set $1,2, \ldots$, $n, \cdots$ may be replaced by a general set of elements $q$, in which there is a transitive order relation, and any two elements have a common successor. However the boundedness of $L_{q}(\xi)$ has a certain uniform character, in that there exists a sequence $\left\{q_{n}\right\}$ independent of $\xi$ such that for each $\xi$ the $L_{q}(\xi)$ are bounded after a certain $q_{n} . \dagger$ Then the set $L_{q}(\xi)$ is ultimately uniformly limited. $\neq$

The definition of bilinear and $n$-linear limited operations is almost self-evident, a form $B\left(\xi_{1}, \xi_{2}\right)$ being bilinear on the classes $\Im_{1}$ and $\Im_{2}$, if it transforms every element $\left(\xi_{1}, \xi_{2}\right)$ of $\left(\widetilde{S}_{1}, \widetilde{S}_{2}\right)$ into a real number, and is linear on $\widetilde{\Im}_{1}$ and $\Im_{2}$ separately; it is limited if there exists an $M$ such that for every $\xi_{1}$ and $\xi_{2}$

$$
\left|B\left(\xi_{1}, \xi_{2}\right)\right| \leqq M\left\|\xi_{1}\right\| \cdot\left\|\xi_{2}\right\| \text {. }
$$

The spaces $\Im_{1}$ and $\Im_{2}$ do not need to coincide, and consequently the symbol || || may have different interpretations.

The question of the general form of a limited bilinear operation on two given spaces has not received very much consideration, and is somewhat beset with difficulty. $\S$ A slight inroad can be made by noting that if we fix the element $\xi_{2}$ of $\widetilde{S}_{2}$, then $B\left(\xi_{1}, \xi_{2}\right)$ is a linear limited operation on $\mathfrak{S}_{1}$ and conversely. This gives results for the cases of Group I, where the elements $\xi$ are sequences, and where the general linear limited operation is known. For instance, if $\mathfrak{S}_{1}=\widetilde{S}_{2}=\mathrm{I} \cdot 1_{1}=$ Hilbert space, then

$$
B\left(\xi_{1}, \xi_{2}\right)=\sum_{p} \sum_{q} x_{p}^{(1)} a_{p q} x_{q}^{(2)}=\sum_{q} \sum_{p} x_{p}^{(1)} a_{p q} x_{q}^{(2)},
$$

complete conditions on $a_{p q}$ in addition to $\sum_{p}\left|a_{p q}\right|^{2}<\infty$ and $\sum_{q}\left|a_{p q}\right|^{2}<\infty$ which make $B$ limited being still undetermined. $\uparrow$

$\dagger$ This has an analogue in the sequential limit. The need of such a condition appears from examples given by von Neumann, Mathematische Annalen, vol. 102 (1929), p. 380.

$\ddagger$ See Hildebrandt, this Bulletin, vol. 29 (1923), p. 311.

$\S$ But see Fréchet, Transactions of this Society, vol. 16 (1915), pp. 216234; and Radon, Wiener Berichte, vol. 122 (IIa) (1913), pp. 1381, etc.

I See Hellinger and Toeplitz, Encyklopädie der Mathematischen Wissenschaften, vol. IIc 13, p. 1426. 
If $\Im_{1}=\Im_{2}=\mathrm{I} .1_{e}$ with $e=0$, then $B$ has the same form as above, the $a_{p q}$ being subject to the condition of being uniformly bounded ( $\leqq M$, the modulus of the operation). The situation is more complicated in the cases of Group II or a combination of the two groups.

By a repeated application of the generalized theorem on sets of linear limited operations, we can get a corresponding theorem on sets of bilinear limited operations, the well known theorem of Toeplitz $\dagger$ which states that if the bilinear form $\sum_{p} \sum_{q} x_{p} a_{p q} y_{q}$ converges for every $\xi$ and $\eta$ of Hilbert space for which $\|\xi\|$ $=\|\eta\|=1$, then it is limited, being a corollary of this theorem by a consideration of the bilinear operations

$$
B_{m n}(\xi, \eta)=\sum_{p=1}^{m} \sum_{q=1}^{n} x_{p} a_{p q} y_{q} .
$$

4. Linear Limited Transformations. A transformation $T$ will be called a linear limited transformation on $\mathfrak{S}_{1}$ to $\mathfrak{S}_{2}$ if it satisfies the following conditions:

(a) To every element $\xi$ of $\Im_{1}$ there corresponds an element $\eta$ of $\widetilde{S}_{2}$.

(b) For every $\xi_{1}$ and $\xi_{2}$ of $\mathfrak{\Im}_{1}$ and all complex numbers $a_{1}$ and $a_{2}$

$$
T\left(a_{1} \xi_{1}+a_{2} \xi_{2}\right)=a_{1} T\left(\xi_{1}\right)+a_{2} T\left(\xi_{2}\right) .
$$

(c) There exists a number $M$ such that for all $\xi$ of $\Im_{1}$

$$
\|T(\xi)\| \leqq M\|\xi\| \text {. }
$$

The smallest possible value of $M$ in (c) will be called the modulus $(M(T))$ of $T$. Obviously the norm for $\Im_{1}$ and $\Im_{2}$ need not be the same. As in the case of operations, condition (c) is equivalent to continuity. Also continuity at a single point is sufficient, with linearity (b), to guarantee continuity at all points and hence limitedness.

This concept covers a rather wide variety of notions, because of the generality of $\Im_{1}$ and $\mathfrak{\Im}_{2}$. For instance, a linear limited operation is a transformation, the space $\widetilde{S}_{2}$ being the set of all complex numbers. A sequence of linear limited operations on $\mathfrak{\varsigma}$ converging for each member of $\mathfrak{S}$ can be considered a linear

† Mathematische Annalen, vol. 69 (1910), pp. 321-322. 
limited transformation on $\subseteq$ to space I.3, which classes certain types of summability definitions as special instances of linear limited transformations. $\dagger$

If the transformation $T$ is not defined on the entire space $\mathfrak{S}$, but only on a fundamental set $\$$, then it can be extended to a linear limited transformation on $\mathfrak{S}$, if, and only if, there exists an $M$ such that for every finite subset $\xi_{1}, \cdots, \xi_{n}$ of $\$$, and every set of numbers $a_{1}, \cdots, a_{n}$, we haveł

$$
\left\|\sum_{i=1}^{n} a_{i} T\left(\xi_{i}\right)\right\| \leqq M\left\|\sum_{i=1}^{n} a_{i} \xi_{i}\right\| .
$$

The question what form a linear limited transformation must take, is in some cases rather difficult to answer. The best chance for success is in the case in which the space $\Im_{2}$ is a set of functions, in which the existence of a norm has as consequence the boundedness of the functional values. In such cases, the values of $\eta(p)$, for $p$ fixed, form a linear limited operation on $\mathfrak{S}_{1}$. For example, if $\widetilde{S}_{2}$ is any of the classes of Group I, the values $\eta(p)$ are certain sequences of linear limited operations. (a) On I.1 to $\mathrm{I} .1_{1}$

$$
y_{p}=\sum_{q} a_{p q} x_{q},
$$

$a_{p q}$ being coefficients of a limited bilinear form. (b) On I.1 $1_{0}$ to I. $1_{1}$ the transformation takes the same form, but the conditions on $a_{p q}$ are that the sums $\sum_{q}\left|a_{p q}\right|^{2}$ shall be uniformly bounded. (c) On II. $1_{1}$ to I. $1_{1}$, a $T$ is of the form $\int f_{p}(q) x(q)$, where the $f_{p}$ are of Lebesgue integrable square, and such that for every $x(q)$ of the same class we have $\sum_{p}\left|\int f_{p} x\right|^{2}<\infty$. What this requires of the sequence $f_{p}$ has been developed by A. J. Pell. $\S$ A sufficient but not necessary condition is that the $f_{p}$ form a normed orthogonal system. (d) On II.1 $1_{1}$ to I.4 a $T$ is of the form as in (c), where the integrals $\int f_{p} x$ form a uniformly bounded sequence for each $\xi$ of II.1. For this it is necessary and sufficient that $\int\left|f_{p}\right|^{2}$ form a bounded sequence. (e) A $T$ on II.3 to II.3 is of the form $\int x(p) d \alpha(p, q)$, where $\alpha$ is of bounded variation for

† See Schur, Journal für Mathematik, vol. 151 (1920), p. 79; Hahn, loc. cit., has given extended consideration of the conditions to be satisfied by special forms of operations so as to be transformations of the type mentioned.

‡ See Hahn, Journal für Mathematik, vol. 157 (1927), p. 216.

\& Transactions of this Society, vol. 12 (1911), p. 142. 
every $q$, as a matter of fact must be uniformly of bounded variation in $p$. In addition it must satisfy certain continuity conditions. The case when $T$ is on II. $1_{1}$ to II. $1_{1}$ is most easily expressed in terms of the indefinite integrals of the functions involved and Hellinger integrals. $\dagger$

It is possible to set up an algebra of linear limited transformations. For instance, if $T_{1}$ and $T_{2}$ are on $\Im_{1}$ to $\Im_{2}$, then $c_{1} T_{1}+c_{2} T_{2}$ is defined as $T_{1}\left(c_{1} \xi_{1}\right)+T_{2}\left(c_{2} \xi_{2}\right)$. Obviously $M\left(c_{1} T_{1}+c_{2} T_{2}\right)$ $\leqq\left|c_{1}\right| M\left(T_{1}\right)+\left|c_{2}\right| M\left(T_{2}\right)$. If we consider the totality of all limited linear transformations on $\mathfrak{S}_{1}$ to $\mathfrak{S}_{2}$, then this can be considered as a vector space of the same type as $\mathfrak{S}$, the norm being the modulus $M(T)$.

If $T_{1}$ is a linear limited transformation on $\widetilde{S}_{1}$ to $\widetilde{S}_{2}$, and $T_{2}$ on $\mathfrak{\Im}_{2}$ to $\mathfrak{\Im}_{3}$, then the succession $T_{1} T_{2}$ will define a linear limited transformation on $\mathfrak{\Im}_{1}$ to $\mathfrak{\Im}_{3}$, and it is obvious that the modulus $M\left(T_{1} T_{2}\right)$ satisfies the condition

$$
M\left(T_{1} T_{2}\right) \leqq M\left(T_{1}\right) M\left(T_{2}\right) .
$$

Obviously $T_{1}$ and $T_{2}$, even when $\Im_{1}=\Im_{2}=\Im_{3}$, are not necessarily commutative. It follows from the definitions and linearity that $T_{1}\left(T_{2}+T_{2}^{\prime}\right)=T_{1} T_{2}+T_{1} T_{2}^{\prime}$, and that three operations $T_{1}, T_{2}, T_{3}$ are associative, that is, $\left(T_{1} T_{2}\right) T_{3}=T_{1}\left(T_{2} T_{3}\right)$.

Of prime interest is the study of reciprocal relations, that is, answering the questions (a) under what conditions does there exist, for a given $\eta$ of $\Im_{2}$, a $\xi$ of $\Im_{1}$ such that $T \xi=\eta$; and more generally (b) when is this possible for every $\eta$ of class $\mathfrak{S}_{2}$ ? The latter is closely allied with the question under what conditions does there exist for a given $T$ on $\mathfrak{S}_{1}$ to $\mathfrak{S}_{2}$ a linear limited transformation $T^{-1}$ on $\mathfrak{S}_{2}$ to $\mathfrak{S}_{1}$ such that $T^{-1} T=I$ and $T T^{-1}=I$, the identity transformation $I$ being on $\widetilde{S}_{1}$ to $\widetilde{S}_{1}$ in the first instance, and on $\mathfrak{S}_{2}$ to $\mathfrak{S}_{2}$ in the second. If the first of these equations is satisfied we call $T^{-1}$ a left hand reciprocal and if the second, then $T^{-1}$ is a right hand reciprocal. It is obvious that if $T$ has a right hand reciprocal $T_{r}^{-1}$, then $\xi=T_{l}^{-1} \eta$ is a solution of $T \xi=\eta$; if $T$ has a left hand reciprocal $T_{r}^{-1}$, then a solution of $T \xi=\eta$, if there exists one, is expressible in the form $\xi=T_{l}^{-1} \eta$. The following results are well known and find their parallel in the theory of groups.

† See Radon, loc. cit., p. 1384. 
[April,

(a) If $T$ has both a right hand and a left hand reciprocal, then they are identical and are the only reciprocal of $T$.

(b) If $T$ has a unique left (or right) hand reciprocal, then this is also a right (or left) hand reciprocal, and it is unique. For if $T^{-1}$ is a left hand reciprocal, then $T^{-1}+S T T^{-1}-S$, for every linear limited $S$ on $\widetilde{S}_{2}$ to $\Im_{1}$, is also one, and so $T T^{-1}=I$.

(c) If there is more than one left (or right) hand reciprocal, then there are an infinite number, any linear combination $c_{1} T_{1}^{-1}+c_{2} T_{2}^{-1}$, with $c_{1}+c_{2}=1$, satisfying this condition.

As a consequence we can make the following statements about reciprocals: (1) either there is a unique right hand, or unique left hand reciprocal, and then there is a reciprocal; or (2) there is no right (left) hand reciprocal and an infinite number of left (right) hand reciprocals; or (3) there is neither a left nor a right hand reciprocal.

Obviously the existence of a reciprocal is not essential to the determination of a $\xi$ for a special $\eta$ such that $T \xi=\eta$.

The convergence of sequences of linear limited transformations may be defined in two ways. (a) For every $\xi$ we have $\lim _{n}\left\|T_{n}(\xi)-T(\xi)\right\|=0$. By applying the theorem of $\S 2$ on sequences of linear operations to $\left\|T_{n}(\xi)\right\|$, it follows that the moduli $M\left(T_{n}\right)$ form a bounded set, and it is obvious that the modulus $M(T)$ is less than or equal to the least upper bound of $M\left(T_{n}\right)$. (b) $\lim T_{n}=T$ may be defined as equivalent to $\lim _{n} M\left(T_{n}-T\right)=0$. It is obvious that in this case we have also $\lim _{n} T_{n}(\xi)=T(\xi)$ for every $\xi$, and $\lim _{n} M\left(T_{n}\right)=M(T)$. If a distinction is necessary one might call the limit in terms of moduli a strong convergence, and the convergence of $T_{n}(\xi)$ to $T(\xi)$ for every $\xi$ a weak convergence. By using the same type of limit throughout one gets the usual theorems on sums and products of sequences.

Of special interest are the transformations which transform the space $\subseteq$ into $\subseteq$ or a subset of $\subseteq$. In that case, it is possible to consider in addition to $T$ the transformations $T^{2}, T^{3}$, etc., and their linear combinations. In particular we can discuss sequences of transformations defined symbolically by $\sum_{n=1}^{\infty} a_{n} T^{n}$, where the $a_{n}$ 's are complex numbers. If the infinite power series $\sum_{n=1}^{\infty}\left|a_{n}\right| \lambda^{n}$ converges for $\lambda=1 / M(T)$, then $\sum_{n=1}^{m} a_{n} T^{n}$ converges in the strong sense to the transformation $\sum_{n=1}^{\infty} a_{n} T^{n}$ and defines a linear limited transformation. Thus we can obtain 
transformations analogous to various analytic functions. For example, $\sum_{n=0}^{\infty} \lambda^{n} T^{n},\left(T^{0}=I\right)$, corresponds to $1 /(1-\lambda x)$ and is a linear limited transformation for $|\lambda|<1 / M(T) ; \sum_{n=0}^{\infty} \lambda^{n} T^{n} /(n !)$ corresponds to $e^{\lambda x}$ and gives us a symbolic solution of the linear system of differential equations $d(\xi(\lambda)) / d \lambda-T(\xi(\lambda))=0$; $\sum_{n=1}^{\infty} \lambda^{n} T^{n} / n$ corresponds to $\log (1-\lambda x)$, and so on.

Applying these ideas to the transformation $I-\lambda T$ and denoting by $I-\lambda T_{\lambda}$ the reciprocal of this transformation for those values of $\lambda$ for which it exists, we find that this reciprocal exists at least for $|\lambda|<1 / M(T)$ and

$$
T_{\lambda}=-\sum_{n=1}^{\infty} \lambda^{n-1} T^{n} .
$$

Assuming that $T_{\lambda}=-T$ for $\lambda=0$, we observe that the relation between $T$ and $T_{\lambda}$ is given by

$$
T+T_{\lambda}=\lambda T T_{\lambda}=\lambda T_{\lambda} T,
$$

the understanding being that the equality holds for all members of $\Im$. Obviously for any two numbers $\lambda$ and $\mu$ for which $T_{\lambda}$ and $T_{\mu}$ exist, it is true that

$$
T_{\mu}-T_{\lambda}=(\lambda-\mu) T_{\lambda} T_{\mu},
$$

which includes the preceding relations for $\lambda=0$ and $\mu=0$, respectively. It follows at once from this relation that the set of points $\lambda$ of the complex plane for which the reciprocal $T_{\lambda}$ exists form an open set. For if $T_{\lambda_{0}}$ exists, then

$$
T_{\mu}=\sum_{n=1}^{\infty}\left(\lambda_{0}-\mu\right)^{n-1} T_{\lambda_{0}}^{n}
$$

will satisfy these relations provided $\left|\mu-\lambda_{0}\right| M\left(T_{\lambda_{0}}\right)<1$. Obviously $T_{\mu}$ is a continuous function of $\mu$ in the space of linear limited transformations. These identities give a more general result, namely, if $\lambda_{1}, \cdots, \lambda_{n}, \cdots$ is a sequence of values converging to $\lambda_{0}$, such that $T_{\lambda_{n}}$ exists for every $n$, and if $M\left(T \lambda_{n}\right)$ are bounded, then $T_{\lambda_{0}}$ exists and $M\left(T_{\lambda_{0}}-T_{\lambda_{n}}\right)$ approaches zero.

On account of the fact that $T_{\lambda}$ has the properties of a holomorphic function in the complex $\lambda$ plane, Riesz $\dagger$ suggests the formation of $\int f(\lambda) T_{\lambda}$ along continuous curves in the $\lambda$ plane lying in

$\dagger$ Riesz, Inf. Inc., p. 118. 
regions of existence of $T_{\lambda}$, as transformations related to $T_{\lambda}$. He uses with success the transformations $\int T_{\lambda} \lambda^{k} d \lambda$ along curves not containing the origin, to separate $T$ into orthogonal sections similar to the decomposition of the kernel of a linear integral equation relative to the singularities of the reciprocal. The method seems worthy of further study.

5. Completely Continuous Transformations. The discussion of the singularities of the transformation $T_{\lambda}$ and their influence on the transformation $T$ in case $T$ is limited but otherwise unrestricted is somewhat difficult. It is possible, however, to obtain results comparable to those of linear integral equations if the transformation $T$ is restricted to be completely continuous. While a linear limited, and so continuous, transformation transforms a bounded sequence into a bounded sequence and a compact sequence into a compact sequence, a completely continuous transformation is defined $\dagger$ to be one which transforms bounded sequences into compact sequences. It is obvious that the identity transformation is not completely continuous unless the space $\mathfrak{S}$ is of finite dimension. If we assume for convenience that our transformations are on $\subseteq$ to $\subseteq$, then if $T$ and $S$ are two transformations of which one is completely continuous the composites $T S$ and $S T$ are completely continuous, so that the iterates $T^{2}, T^{3}, \cdots$ of $T$ are completely continuous if $T$ is. It follows further that, excepting in a space of finite dimension, a completely continuous transformation cannot have a reciprocal. Also if $T_{n}$ is a sequence of continuous transformations converging to $T$ in the strong sense, that is, $M\left(T_{n}-T\right)$ approaches zero, then $T$ is also completely continuous, which is not true in general if the approach is in the weak sense. The completely continuous transformations form a complete linear subspace of the space of all linear limited transformations, the norm being the modulus.

The simplest completely continuous transformations are those which transform $\mathfrak{S}$ into a subset of finite dimension. It follows that if $T$ is the strong limit of a sequence of such transforma-

† See Riesz, $L F G$, p. 74. Transforming a compact sequence into a bounded sequence makes $T$ still limited, and so continuous. J. von Neumann, (Mathematische Annalen, vol. 102 (1929), p. 70), has suggested a weaker continuity condition in the notion closed, $T$ being closed if $\lim \xi_{n}=\xi$ and $\lim T \xi_{n}=\eta \mathrm{im}$ plies $T \xi=\eta$. 
tions, then $T$ is completely continuous. Whether the theorem is reversible without additional limitations on the space considered seems to be still undetermined. The converse theorem does hold in the spaces $\mathrm{I} .1_{e}(e \geqq 0), \mathrm{II} .1_{e}(e>0)$, and II.3.

Riesz $\dagger$ has shown that the transformations of the form $I-T$ and $I-\lambda T$, where $T$ is completely continuous and $\lambda$ is a complex number, have properties similar to those of the finite dimensional and Fredholm integral equation case. In particular he has proved the following theorems, mainly on the basis of the fact that for a linear closed set of finite dimension, compactness and boundedness are equivalent.

A. Either the functional equation $\xi-T \xi=\eta$ has a solution $\xi$ for each $\eta$ of $\mathfrak{S}$ or there exist non-zero solutions of $\xi-T \xi=0$, the number of linearly independent solutions being finite.

B. There exists a finite integer $N$ such that every solution of $(I-T)^{n} \xi=0$ for $n>N$ is a solution of $(I-T)^{N} \xi=0$, while $(I-T)^{N} \xi=0$ has solutions not present if $n<N$.

C. The transformation $I-T$ transforms $\subseteq$ into a linear subclass $\mathfrak{\Im}_{1}$ of itself. For the same $N$ as in $\mathrm{B}$, the class $\mathfrak{S}_{n}=\Im_{N}$ for $n>N$, but $\mathfrak{\Im}_{n}>\mathfrak{\Im}_{N}$ for $n<N$.

D. There exists a separation of $T$ into two transformations $T_{1}, T_{2}$ which are orthogonal, that is, $T_{1} T_{2}=T_{2} T_{1}=0$, such that $\left(I-T_{1}\right)\left(I-T_{2}\right)=I-T ; I-T_{2}$ has a reciprocal, $I-T_{1}$ has the same characteristic elements as $T$.

It is possible to parallel even the adjointness properties as in the Fredholm theory, by noting that the solutions of the homogeneous equation $(I-T) \xi=0$ can be considered as a transformation $T_{0}$ on $\sqrt{ }$ to the set of solutions $T_{0}$ satisfying the conditions

$$
(I-T) T_{0}=\left(I-T_{0}\right) T_{0}=0 .
$$

It can be shown that there exists a transformation $T_{0}{ }^{*}$ on $\mathfrak{S}$ to a set of finite dimension, the maximum dimension being the same as that belonging to $T_{0}$, such that $\ddagger$

$$
T_{0}^{*}\left(I-T_{0}^{*}\right)=T_{0}^{*}(I-T)=0 .
$$

For the reciprocal $I-\lambda T_{\lambda}$ of $I-\lambda T$, the singular points are

$\dagger L F G$, pp. 79, etc.

‡ See Hildebrandt, Acta Mathematica, vol. 51 (1928), pp. 311-318. 
isolated in the complex plane. It can be shown that the transformation $T_{\lambda}$ has an expansion of the form

$$
T_{\lambda}=\sum_{-N}^{\infty} T_{n}\left(\lambda-\lambda_{0}\right)^{n}
$$

valid in a neighborhood of the point $\lambda_{0}$, the integer $N$ being identical with the $N$ of properties $\mathrm{B}$ and $\mathrm{C}$ above, the transformations $T_{n}$ for $n<0$ being iterates of a transformation $S$ transforming $\subseteq$ into a linear subspace of finite dimension, thus paralleling the elementary divisor theory.

If now $\lambda_{1}, \lambda_{2}, \cdots$ are the singular points of $T_{\lambda}$, we can multiply $T_{\lambda}$ by an entire function $D(\lambda)$ having $\lambda=\lambda_{n}$ as roots of order $N_{n}$. The transformation $D(\lambda) \cdot T_{\lambda}$ will have no singularities in the finite part of the $\lambda$ plane, and consequently be expressible in the form $\sum_{i=1}^{\infty} T_{i} \lambda^{i}$, that is, in essence, $T_{\lambda}$ is the quotient of two entire functions, as in the Fredholm theory.

It is possible to extend these results in a number of directions.

(a) Consider a transformation expressed in the form $S-\lambda T$, where $S$ has a reciprocal $S^{-1}$. Then if the reciprocal of $S-\lambda T$ exists and is written in the form $S^{-1}-\lambda T_{\lambda}$, the transformation $T_{\lambda}$ satisfies reciprocal relations of the form

$$
S^{-1} T+T_{\lambda} S-\lambda T_{\lambda} T=T S^{-1}+S T_{\lambda}-\lambda T T_{\lambda}=0,
$$

which reduce to the usual relations for $S=S^{-1}=I$. If further $T$ is completely continuous, then the properties of $S-\lambda T$ are similar to those of $I-\lambda T$ discussed above.

(b) Returning to the transformation $I-\lambda T$, we see that it possesses a reciprocal at least for $\lambda<1 / M(T)$. Let $\epsilon$ be the greatest lower bound of values $\epsilon_{1}$ for which there exists a completely continuous transformation $S_{\epsilon_{1}}=S$ such that $M(T-S)<\epsilon_{1}$. If now we write

$$
I-\lambda T=I-\lambda(T-S)+\lambda S,
$$

then $I-\lambda(T-S)$ has a reciprocal for $|\lambda|<1 / \epsilon$. Hence in the circle $|\lambda|<1 / \epsilon$ we have a condition similar to the case where $T$ is completely continuous. In particular, there exists a power series $D(\lambda)$ and an expansion $\sum \lambda^{n} T_{n}$ such that $D(\lambda) T_{\lambda}=\sum \lambda^{n} T_{n}$, the expansions being valid for $|\lambda|<1 / \epsilon$. If $\epsilon=0$ we have the Fredholm or completely continuous case. This is in essence a 
generalization of the Schmidt "Abspaltungsverfahren" $\dagger$ the approximating transformation $S$ being completely continuous instead of finite dimensional. $\ddagger$

6. Adjoint Spaces and Adjoint Linear Transformations. In the theory of linear integral equations the adjoint equation, suitably defined, plays an important role. Since on our general range the explicit form of a transformation is not given, it seems a difficult matter to formulate an adjoint theory. Examination of existing instances would indicate that the definition of adjoint transformations might be based on the notion of adjoint spaces, which in turn depends upon the correspondence set up between spaces by linear operations. We consequently formulate, with Hahn, $\S$ the following definition.

The adjoint space $\mathfrak{I}$ of any linear complete vector space $\mathfrak{S}$ consists of the totality of all linear limited operations on $\mathbb{S}$.

It is obvious that the space $\mathfrak{I}$ is linear. If we take as norm the modulus of the operation, then the norm satisfies the triangle property, and the space is complete. If we denote elements of $\mathfrak{I}$ by $\eta$, then every linear limited operation on $\mathfrak{S}$ is expressible in the form

$$
L(\xi)=(\eta, \xi)
$$

where $(\eta, \xi)$ is a bilinear operation on $\mathfrak{T} \subseteq$ whose modulus is unity. Obviously, for fixed $\xi,(\eta, \xi)$ is a linear operation on $\mathfrak{T}$.

The adjoint space of any given space may be realized in more than one way, but the different realizations are equivalent in the sense that there exists a one to one correspondence between them, that is, if $\mathfrak{I}_{1}$ and $\mathfrak{I}_{2}$ are adjoint to $\mathfrak{S}$, then there exists a linear limited transformation on $\mathfrak{I}_{1}$ to $\mathfrak{I}_{2}$ with a reciprocal.

As examples of adjointness, we note the following. The Hil-

† For a similar procedure, see Radon, Wiener Berichte, vol. 128 IIa (1919), pp. 1106-1114; Hille and Tamarkin, Annals of Mathematics, vol. 31 (1930), p. 509.

$\ddagger$ We might mention that, while the first general theory of integral equations of E. H. Moore (this Bulletin, vol. 28 (1912), pp. 334-362) does not seem to be a special case under the above developments, still he treats completely continuous transformations, particularly of the type which can be uniformly approximated by transformations of finite dimension. The non-determinantal results can in fact be derived, following Schmidt, with fewer postulates than are sufficient to make Fredholm determinants available.

§ Journal für Mathematik, vol, 157 (1927), p. 218. 
bert space $I .1_{1}$ and $\Omega^{2}$ space II. $1_{1}$ are self-adjoint; the adjoints of I.1 $1_{e}$ and II.1 $1_{e}(e>0)$ are I. $1_{1 / e}$ and II. $1_{1 / e}$, respectively; the adjoint of I. $1_{0}$ is I.4, of I. $3_{0}$ is I. $1_{0}$, and so on.

The adjoint of the adjoint space $\mathfrak{I}$ of $\mathfrak{S}$ while including $\mathfrak{S}$ may be larger. Thus the adjoint of I. $3_{0}$ is I.1 $1_{0}$ of I.1 $1_{0}$ is I.4 and so on. How often this process of taking adjoints may be repeated is still undetermined. Hahn calls a space where the adjoint of $\mathfrak{I}$ is $\subseteq$ regular. I. $1_{e}$ and II.1 $1_{e}(e>0)$ are regular.

The notion of adjoint space makes possible the definition of a weak limit in $\mathfrak{S}$. Thus weak limit $\xi_{n}=\xi$ may be defined as

$$
\lim \left(\eta, \xi_{n}\right)=(\eta, \xi) \text { for every } \eta \text { of } \mathfrak{T} \text {. }
$$

Since the adjoint of $\mathfrak{I}$ may be other than $\mathfrak{S}$, the definition of a weak limit in $\mathfrak{I}$, namely,

weak limit $\eta_{n}=\eta$, equivalent to $\lim \left(\eta_{n}, \xi\right)=(\eta, \xi)$ for every $\xi$, would in general be still weaker. One could write down a weak limit relative to a complete subclass $\mathfrak{I}_{0}$ of $\mathfrak{T}$, the $\eta$ ranging over this class only. It is obvious that if weak $\lim \xi_{n}=\xi$, then $\left\|\xi_{n}\right\|$ form a bounded sequence. If the space $\mathfrak{S}$ is the adjoint of $\mathfrak{I}_{0}$ and is separable, then by applying the theorem $\dagger$ on the compactness of sets on the denumerable range, it is possible to show that every bounded subset of $\mathbb{S}$ is weakly compact.

With the aid of the bilinear operation $(\eta, \xi)$ it is possible to define the notion of adjoint transformation. If $T$ is a linear limited transformation on $\mathfrak{S}_{1}$ to $\mathfrak{S}_{2}$ and $\eta_{2}$ any element of $\mathfrak{S}_{2}$, the adjoint of $\Im_{2}$, then $\left(\eta_{2}, T\left(\xi_{1}\right)\right)$ defines a linear limited operation of $\mathfrak{S}_{1}$, and is consequently expressible in the form $\left(\eta_{1}, \xi_{1}\right)$, where $\eta_{1}$ belongs to $\mathfrak{I}_{1}$. We have thus defined a transformation $T^{*}$ on $\mathfrak{I}_{2}$ to $\mathfrak{I}_{1}$, which is obviously linear and limited and has the same modulus as $T$. The adjoint of $T^{*}$ is $T$ if $\mathfrak{S}_{1}$ is regular, otherwise it agrees with $T$ on $\mathfrak{S}_{1}$, but may be on a space including $\widetilde{S}_{1}$ to a space including $\mathfrak{S}_{2}$.

If $T_{1}$ and $T_{2}$ have as adjoints $T_{1}{ }^{*}$ and $T_{2}{ }^{*}$, then the adjoint of $T_{1} T_{2}$ is $\dot{T}_{2}{ }^{*} T_{1}{ }^{*}$. Further the adjoint of the identity is the identity. Hence if $T$ has a reciprocal, the adjoint $T^{*}$ also has a reciprocal, namely, $\left(T^{-1}\right)^{*}$.

If $T$ transforms the space $\subseteq$ into a space of finite dimensions,

† See Fréchet, Rendiconti di Palermo, vol. 26 (1906), p. 42. 
the adjoint $T^{*}$ on $\mathfrak{I}$ has the same property, the dimensions of the space being the same for both.

If the space $\mathfrak{S}$ is separable, then the adjoint of a completely continuous transformation on $\subseteq$ to $\subseteq$ is again completely continuous. Whether this holds in general, is undetermined. However if $T$ is completely continuous, then the transformation $I-\lambda T^{*}$ has properties similar to those of $I-\lambda T$. In particular, the transformation $I-\lambda T^{*}$ has a reciprocal if and only $I-\lambda T$ has, the equation $I-\lambda T^{*}=0$ has as many linearly independent solutions as $I-\lambda T=0$, and the reciprocal $I-\lambda T_{\lambda}{ }^{*}$ of $I-\lambda T^{*}$ is expressible in the form

$$
\left(\sum_{i=0}^{\infty} d_{i} \lambda^{i}\right) T_{\lambda}^{*}=\sum_{i=0}^{\infty} T_{i}^{*} \lambda^{i}
$$

where $\sum d_{i} \lambda^{i}$ is a permanently convergent power series, and $\sum \lambda^{i} T_{i}{ }^{*}$ converges for all values of $\lambda$ in the modular sense, the $T_{i}{ }^{*}$ being the adjoints of the transformations corresponding to $T_{\lambda}$.

It is a simple matter to prove the following results due to Riesz:

(a) in order that for a given $\xi_{2}$ of $\mathfrak{S}_{2}$ there exist a $\xi_{1}$ of $\mathfrak{\Im}_{1}$ such that

$$
T\left(\xi_{1}\right)=\xi_{2},
$$

it is necessary that there exist a constant $M$ such that for every $\eta_{2}$ of $\mathfrak{I}_{2}$

$$
\left|\left(\eta_{2}, \xi_{2}\right)\right| \leqq M\left\|T^{*}\left(\eta_{2}\right)\right\| ;
$$

and (b) in order that $T$ have a reciprocal, it is necessary that there exist an $M$ such that for every $\eta_{1}$ of $\mathfrak{I}_{1}$ and $\xi_{1}$ of $\mathfrak{S}_{1}$

$$
\left|\left(\eta_{1}, \xi_{1}\right)\right| \leqq M\left\|T\left(\xi_{1}\right)\right\| \cdot\left\|\eta_{1}\right\|
$$

and for every $\eta_{2}$ of $\mathfrak{T}_{2}$ and $\xi_{2}$ of $\mathfrak{S}_{2}$

$$
\left|\left(\eta_{2}, \xi_{2}\right)\right| \leqq M\left\|T^{*}\left(\eta_{2}\right)\right\| \cdot\left\|\xi_{2}\right\| \text {. }
$$

Riesz and Radon $\dagger$ have shown that these conditions are also sufficient in special cases, characterized, perhaps, by the fact

† See Riesz, Mathematische Annalen, vol. 69 (1910), pp. 469 etc.; Annales de l'École Normale, (3), vol. 28 (1911), pp. 33-62; Riesz, Inf. Inc., Chap. IV; Radon, Wiener Berichte, vol. 122, IIa (1913), pp. 1386, etc.; Helly, Monatshefte, vol. 31 (1921), pp. 60-91. 
that the spaces $\mathfrak{S}_{1}$ and $\mathfrak{S}_{2}$ are separable, and have regular adjoint spaces.

7. Hilbert or Hermitian Spaces. Hermitian Transformations. $\dagger$ The derivation of more detailed results on the singularities of the transformations $T$ and $I-\lambda T$ is hampered by a lack of symmetry in the general vector space which forms the basis of the preceding sections. Limiting the spaces to which our considerations apply by introducing an element of symmetry into the bilinear operation defining adjoint spaces makes it possible not only to obtain a larger body of results for limited transformations, but even to consider special types of transformations, unlimited, or defined only on a portion of the space. The principal additional conditions as given by J. V. Neumann $\ddagger$ and M. H. Stone $\S$ on the space are in connection with condition B on our vector space. The norm $\|\xi\|$ is defined in terms of a bipartite operation $(\xi, \eta)$ on the space to complex numbers, subject to the Hermitian condition

$$
(\xi, \eta)=(\overline{\eta, \xi}),
$$

linear in the first element and so conjugately linear in the second, and such that for every $\xi,(\xi, \xi) \geqq 0$, zero only if $\xi=0$. Then by definition

$$
\|\xi\|^{2}=(\xi, \xi),
$$

and this satisfies the conditions on norm. In addition it is assumed (D) that the space is separable, that is, a denumerable set is dense in the space. A complete vector space satisfying these conditions if of infinite dimensions is called a Hilbert space. Due to the fact that after all the Hermitian operator $(\xi, \eta)$ is central, it seems more to the point to call it a Hermitian

$\dagger$ The principal references for this section are the following: HellingerToeplitz, Encyklopädie der Mathematischen Wissenschaften, vol. IIC, p. 1575; Riesz, Inf. Inc., Chapters IV and V; E. H. Moore, Mathematische Annalen, vol. 86(1922), pp. 34;T. Carleman, Sur les équations intégrales singulières, Upsala Arsskrift, 1923; J. v. Neumann, Mathematische Annalen, vol. 102 (1929), pp. 49-137, 370-427; M. H. Stone, Proceedings of the National Academy, vol. 15 (1929), pp. 198-200, 423-5; A. Wintner, Spektraltheorie der unendlichen Matrizen, Leipzig, 1929; F. Riesz, Szeged Acta Litterarum, vol. 5 (1930), pp. 23-54; this will be cited in what follows as Szeged Acta.

$\ddagger$ Loc. cit., p. 64 .

§ Loc. cit., p. 198. 
space, reserving the name Hilbert space for the space $\mathrm{I} .1_{1}$, that is, $\sum\left|x_{n}\right|^{2}<\infty$. In any case we shall denote by $\mathfrak{S}$ a space which is (A) a vector space, (B) such that $\|\xi\|=(\xi, \xi)$, (C) complete, and (D) separable.

The usual instances of such spaces are the Hilbert space I.1 with $(\xi, \eta)=\sum x_{p} \bar{y}_{p}$ and the $\Omega^{2}$ space II. $1_{1}$ with $(\xi, \eta)=\int \xi \bar{\eta}$. E. H. Moore $\nmid$ has given a means of constructing such spaces, not all of which satisfy the separability condition $\mathrm{D}$, however. If $\mathfrak{B}$ is any class of elements and $\epsilon(p, q)$ a complex-valued function satisfying the condition that for any finite set $p_{1}, \cdots, p_{n}$ of elements of $\mathfrak{B}$, the matrix $\epsilon\left(p_{i}, p_{j}\right)$ is the matrix of coefficients of a positive Hermitian form, then the function $\xi(p)$ is a member of the space $\subseteq$ if there exists a constant $N$ such that for every finite set of elements $p_{1}, \cdots, p_{n}$ and for any set of complex numbers $x_{1}, \cdots, x_{n}$ we have

$$
\sum_{i=1}^{n} \bar{x}_{i} \xi\left(p_{i}\right) \sum_{j=1}^{n} x_{j} \bar{\xi}\left(p_{j}\right) \leqq N \sum_{i, j=1}^{n} \bar{x}_{i} \epsilon\left(p_{i} p_{j}\right) x_{j}
$$

The greatest lower bound of the possible numbers $N$ is $\|\xi\|^{2}$. The operation $(\xi, \eta)$ is

$$
\lim _{\sigma} \operatorname{det}\left(\begin{array}{cc}
\epsilon\left(p_{i} p_{j}\right) & \xi\left(p_{i}\right) \\
\eta\left(p_{j}\right) & 0
\end{array}\right) \div \operatorname{det} \epsilon\left(p_{i} p_{j}\right)
$$

where $\sigma$ is any set $p_{1}, \cdots, p_{n}$ of elements of $\mathfrak{P}$ for which det $\epsilon\left(p_{i} p_{j}\right)$ is not zero and $\lim _{\sigma} F(\sigma)=a$ is defined; for every $e>0$ there exists a $\sigma_{e}$ such that for any $\sigma$ containing all the points of $\sigma_{e}$ we have $|F(\sigma)-a| \leqq e$. Interesting instances of these spaces are (1) $\mathfrak{B}=[0 \leqq p \leqq 1] ; \epsilon(p, q)=\delta(p q)=0$ for $p \neq q$ and 1 for $p=q ;(2)$ in the space of infinite dimensions, consider a matrix set of elements $a_{p q}$ such that $\sum_{q}\left|a_{p q}\right|^{2}<\infty$; then $\epsilon(p, q)$ $=\sum_{r} \bar{a}_{p r} a_{q r}$ gives a space of interest in connection with the Schmidt $\ddagger$ solution of the system

$$
\sum_{q} a_{p q} x_{q}=y_{p}
$$

for which $\sum\left|x_{p}\right|^{2}<\infty$; (3) the analogue of (2) in $\mathfrak{R}^{2}$ space, namely, $f_{n}(p)$ such that $\int\left|f_{n}\right|^{2}<\infty$ and $\epsilon(n, m)=\int \bar{f}_{n} f_{m} \S ;(4)$ if

$\dagger$ Loc. cit., pp. 34, etc.

¥ See Rendiconti di Palermo, vol. 25 (1908), pp. 53-77; Riesz, Inf. Inc., pp. 65 , etc.

§ See A. J. Pell, loc. cit. 
$\mathfrak{B}$ is an additive class of sets, and $\eta$ is a positive absolutely additive set function on $\mathfrak{P}$, then $\epsilon(p, q)=\epsilon\left(E_{1}, E_{2}\right)=\eta\left(E_{1} \cdot E_{2}\right)$ will give rise to integrals of the Hellinger-Radon type.

Returning to the space $\mathfrak{S}$, in terms of $(\xi, \eta)$, we have a Schwarz inequality

$$
(\xi, \eta)(\eta, \xi) \leqq(\xi, \xi)(\eta, \eta)=\|\xi\|^{2}\|\eta\|^{2} .
$$

Further it is possible to define orthogonality, to reduce any system of elements to a linearly equivalent system of normed orthogonal elements, necessarily denumerable, and to express any element $\xi$ in terms of a complete system of normed orthogonal elements (that is, one whose linear extension is dense in $\mathfrak{S})$, by the Fourier expansion

$$
\xi=\sum_{n=1}^{\infty}\left(\xi, \phi_{n}\right) \phi_{n},
$$

(convergence in the sense of norms) with

$$
(\xi, \xi)=\sum_{n=1}^{\infty}\left(\xi \phi_{n}\right)\left(\phi_{n} \xi\right)=\sum_{n=1}^{\infty}\left|\left(\xi, \phi_{n}\right)\right|^{2} .
$$

As a consequence it is possible to set up a one to one correspondence between any space $\mathfrak{S}$ and the Hilbert space I.1. $1_{1} \dagger$

Riesz $\ddagger$ has pointed out that the following lemma is still valid even if the separability condition $D$ is not fulfilled:

Lemma I. If $\mathfrak{S}_{0}$ is any subset of $\mathfrak{S}$ which is not dense in $\mathfrak{S}$, then there exists an element $\xi$ of $\mathfrak{S}$, for which $\|\xi\|=1$, which is orthogonal to all the elements of $\mathfrak{S}_{0}$.

By the use of the Fourier expansion it is possible to make the connection between the adjoint operation of the preceding section and $(\xi, \eta)$ as follows:

LEMMA II.§ If $L(\xi)$ is a linear limited operation on $\mathfrak{S}$, or on

$\dagger$ If the separability condition $D$ were dropped, a non-denumerable set of mutually orthogonal functions would be possible, for example, in the Moore instance (1). However any given element would be orthogonal to all but a denumerable subset of these, and the Fourier expansion would still be valid in form. Compare also the situation in almost periodic functions.

$\ddagger$ Szeged Acta, p. 28.

§ See also J. von Neumann, loc. cit., p. 94. 
a subset of $\mathfrak{S}$ dense in $\mathfrak{S}$, then there exists a unique element $\eta$ of Se such that

$$
L(\xi)=(\xi, \eta)
$$

8. Linear Limited Transformations. When it comes to linear limited transformations $T$ on $\mathfrak{S}$ to $\mathfrak{E}$, it is obvious that any complete normed orthogonal system will set up a one to one correspondence between $T$ and a matrix $t_{p q}$, formed from the coefficients of a linear limited transformation on Hilbert space I.1 to the same space. $\dagger$ As a consequence all of the results obtained by Hilbert and his followers for this theory can be translated to the space $\mathfrak{S}$. However, some of the results gain in elegance and lucidity by being derived without using the explicit form of the transformation on the space $\mathfrak{S}$, and point the way to further generalizations.

Since every linear limited operation on $\mathfrak{S}$ is of the form $(\xi, \eta)$ we can ai once define the adjoint of a linear limited transformation $T$ by the existent $T^{*}$ such that for every $\xi$ and $\eta$

$$
(T \xi, \eta)=\left(\xi, T^{*} \eta\right)
$$

If $T=T^{*}$, then $T$ is said to be self adjoint or Hermitian, and we shall denote such a transformation by $H$. Obviously, for any $\xi$, $(H \xi, \xi)$ is a real number. If for all $\xi$ we have $(H \xi, \xi) \geqq 0, H$ is said to be positive, negative being similarly defined. Connected with any limited transformation $T$ are the Hermitian transformations

$H_{1}=T+T^{*}, H_{2}=i\left(T-T^{*}\right), H_{3}=T^{*} T$ and $H_{4}=T T^{*}$.

In terms of $H_{1}$ and $H_{2}$ we obviously have

$$
T=\frac{1}{2}\left(H_{1}-i H_{2}\right) \text {. }
$$

The transformations $H_{3}$ and $H_{4}$ are obviously positive. In case $H_{3}$ and $H_{4}$ are the same, that is, $T T^{*}=T^{*} T$, the transformation $T$ is said to be normal, for which a necessary and sufficient condition is that $H_{1}$ and $H_{2}$ be commutative. If finally $T T^{*}=T^{*} T$ $=I$, then $T$ is the analogue of an orthogonal transformation,

$\dagger$ A similar correspondence is obviously possible if $T$ is on a space $\mathfrak{S}$ to another space $\mathfrak{S}^{\prime}$, in which $\mathfrak{S}$ and $\mathfrak{S}^{\prime}$ might agree except for the fundamental normed orthogonal system used. 
and is called unitary $(U)$. Obviously $\|U \xi\|=\|\xi\|$, that is, $U$ leaves lengths of vectors unchanged.

If $H$ is limited, then the set of values $(H \xi, \xi)$ for $\|\xi\|=1$ is limited to a portion of the real axis: $(m, M)$. For the case of any linear transformation $T$, not necessarily limited, but defined on the entire space $\mathfrak{S}$, Stonet has generalized a theorem of Hausdorff on forms in $n$ variables, to the effect that the set of complex values of $(T \xi, \xi)$ subject to the condition $\|\xi\|=1$ forms a convex set of points in the complex plane.

Toeplitz has shown that if $T$ is a transformation of the form $\sum_{q} t_{p q} x_{q}$ on I.1. $1_{1}$ space to I.1 $1_{1}$ space, and valid for all points of the space, then it is necessarily limited $\ddagger$ In dealing with a transformation on the space $\mathfrak{S}$, assumed to be linear only, it does not follow that it can be realized by a transformation of the form indicated in the space $I .1_{1}$, that is, the Toeplitz theorem is not immediately transferable. In this direction there is a result of J. von Neumann, $\S$ namely, that if $T$ is a Hermitian transformation on all of $\mathfrak{S}$ space and satisfies the weaker closed condition that if $\xi_{n}$ approaches $\xi$ and $T \xi_{n}$ approaches $\eta$, then $T(\xi)=\eta$, it follows that $T$ is limited.

Because of their greater simplicity, the properties of linear limited Hermitian transformations are most elegant and extensive. The following theorem is of particular importance.

THEOREM 2. The transformation $I-\lambda H$ has a reciprocal for all complex and real $\lambda$ exterior to the interval $(m, M)$ of variation of $(H \xi, \xi)$ for $\|\xi\|=1$.

Let $\lambda=\lambda_{1}+i \lambda_{2}$. Then if $\lambda$ is exterior to $(m, M)$, $\|(I-\lambda H) \xi\|^{2}=\|\xi\|^{2}-2 \lambda_{1}(H \xi, \xi)+\left(\lambda_{1}{ }^{2}+\lambda_{2}{ }^{2}\right)\|H \xi\|^{2}>K\|\xi\|^{2}$, where $K$ is a positive number depending on $\lambda$. It follows from this inequality that $(I-\lambda H) \xi=0$ has as unique solution $\xi=0$, and that $(I-\lambda H)$ operating on the space $\mathfrak{S}$ gives a closed subset $\mathfrak{S}_{0}$. The fact that this set is dense on $\mathfrak{S}$ and so agrees with $\mathfrak{S}$ now follows at once from Lemma 1 . For if $\eta$ were such that $\|\eta\|=1$ and were orthogonal to $\mathfrak{S}_{0}$, then $(H \eta, \eta)$ would take on a value $\lambda$ exterior to $(m, M)$.

$\dagger$ This Bulletin, vol. 36 (1930), pp. 259-62.

$\ddagger$ Riesz, Inf. Inc., pp. 78, 82, suggest that this is true for other spaces of Group I and is probably deducible from the general theorem of $\S 3$ above.

§ Loc. cit., p. 107. 
As a consequence of this theorem and the results on reciprocals of $\S 4$, it follows that if $H$ is such that the values of $(H \xi, \xi)$ for $\|\xi\|=1$ are bounded from zero, then there exists a number $k \neq 0$ such that $k H=I-(I-k H)$ and so $H$ has a reciprocal. $\dagger$ An immediate consequence of this is that if, for any limited transformation $T,\left(T T^{*} \xi, \xi\right)$ and $\left(T^{*} T \xi, \xi\right)$ are bounded from zero for $\|\xi\|=1$, then $T T^{*}$ and $T^{*} T$ have reciprocals, and $T$ has a reciprocal, namely,

$$
\left(T^{*} T\right)^{-1} T^{*}=T^{*}\left(T T^{*}\right)^{-1} .
$$

These conditions are also necessary. $\ddagger$

The next result is fundamental. $\S$

TheOREM 3. To every linear limited transformation $H$ there correspond two transformations $E_{-}$and $E_{+}$(Einzeltransformationen) having the following properties:

$$
E_{-} E_{-}=E_{-}, \quad E_{+} E_{+}=E_{+}, \quad I=E_{-}+E_{+} .
$$

(2) $E_{-}$and $E_{+}$are commutative and commutative with any transformation commutative with $H$.

(3) $\mathrm{HE}_{-}$and $\mathrm{HE}_{+}$are respectively negative and positive.

(4) For all $\xi$ such that $H \xi=0$ we have $E_{-} \xi=0$ and $E_{+} \xi=\xi$.

At first sight it might seem that $E_{+} \xi$ could be defined as $\xi$ if $(H \xi, \xi) \geqq 0$ and zero if $(H \xi, \xi) \leqq 0$, and $E_{-} \xi=\xi-E_{+} \xi$. However, the transformations so defined are not necessarily linear, nor do they satisfy condition (2). Instead the path leads through the notion of polynomials in $H$, and via uniformly convergent sequences of polynomials to continuous functions in $H$ corresponding to polynomials and continuous functions on $(m, M)$, positive if the corresponding function is positive and mutually commutative. The transformation $H_{+}=H E_{+}$corresponds to $\frac{1}{2}(t+|t|)$ and $H_{-}=H E_{-}$to $\frac{1}{2}(t-|t|)$. Then $H_{+}$and $H_{-}$are orthogonal. The transformation $E_{-}$is then defined as transforming all of the functions of the set $\left[H_{-} \xi\right]$ into themselves, and the functions orthogonal to these into zero, while we set $E_{+}=I-E_{-}$. If the parallelism between functions and transfor-

$\dagger$ Method due to Hilb; see Riesz, Inf. Inc., pp. 93, etc.

$¥$ See, for instance, Wintner, loc. cit. p. 138.

$\S$ See J. von Neumann, loc. cit., pp. 112-4; Riesz, Szeged Acta, pp. 31-38; Inf. Inc., pp. 135-138. 
mations is extended to certain discontinuous functions, then $E_{\text {_ }}$ corresponds to the function unity for $t<0$ and zero for $t \geqq 0$.

Let now $E(\lambda)$ be the transformation of the type $E_{-}$corresponding to the Hermitian transformation $I-\lambda H, \lambda$ real. These transformations correspond to a fundamental set of characteristic functions. They have the following properties:

(1) $E(\lambda) \xi=0$ if $\lambda<m ; E(\lambda) \xi=I \xi=\xi$ if $\lambda>M$.

(2) $E\left(\lambda_{1}\right) E\left(\lambda_{2}\right) \xi=E\left(\lambda_{1}\right) \xi$ if $\lambda_{1} \leqq \lambda_{2}$ so that $(E(\lambda) \xi$, $\xi)$ is increasing in $\lambda$ for each $\xi$.

(3) $E(\lambda) \xi$ is continuous on the left.

(4) $H \xi=\int_{m}^{M} \lambda d_{\lambda} E(\lambda) \xi, \quad I \xi=\int_{m}^{M} d_{\lambda} E(\lambda) \xi$,

the integral being taken in the sense of Stieltjes extended to vector space and norm convergence. The set $E(\lambda)$ is uniquely determined by the transformation $H$. It is called by Stone a canonical resolution of the identity. Riesz suggests the term spectral set.

The transformations $E(\lambda)$ define the spectrum of $I-\lambda H$, and make available a classification of the points of the interval $(m, M)$. For instance the points $\lambda_{0}$ for which there exists a $\xi$ with $\|\xi\|=1$ and for which $(E(\lambda) \xi, \xi)$ is discontinuous at $\lambda_{0}$ correspond to the point spectrum, which, because of the separability of $\mathfrak{S}$, forms a denumerable set. For such $\lambda_{0}$ the homogeneous equation $\xi-\lambda_{0} H \xi=0$ has a non-zero solution. By subtracting, so to speak, the discontinuities of $E(\lambda)$ one gets the part which gives the continuous spectrum and the differential solutions of the homogeneous equation. $\dagger$

If $f(H)$ corresponds to the continuous function $f(x)$ on $(m, M)$, then

$$
f(H) \xi=\int_{m}^{M} f(\lambda) d_{\lambda} E(\lambda) \xi .
$$

In particular, the notion is extensible to non-Hermitian transformations associated with $H$, and we have for the reciprocal

$$
(I-\mu H)^{-1}=\int_{m}^{M} \frac{d_{\lambda} E(\lambda) \xi}{\lambda-\mu},
$$

† For details, see Riesz, Inf._Inc., pp. 141, etc. 
for the set of values of $\mu$ for which it exists.

Similar results apply to the normal limited transformations $T$, for which $T^{*} T=T T^{*}$, and which are expressible in the form $H_{1}+i H_{2}, H_{1}$ and $H_{2}$ being commutative and Hermitian. If $E_{1}(\lambda)$ corresponds to $H_{1}$ and $E_{2}(\mu)$ to $H_{2}$, then $E_{1}$ and $E_{2}$ are commutative for every $\lambda$ and $\mu$. By utilizing the fact that $I=\int d E(\lambda)$, we have

$$
\begin{aligned}
T=H_{1}+i H_{2} & =\int_{m_{2}}^{M_{2}} \int_{m_{1}}^{M_{1}}(\lambda+i \mu) d_{\lambda \mu} E_{1}(\lambda) E_{2}(\mu) \\
& =\int_{m_{1}}^{M_{1}} \int_{m_{2}}^{M_{2}}(\lambda+i \mu) d_{\mu \lambda} E_{2}(\mu) E_{1}(\lambda),
\end{aligned}
$$

where $\Delta_{\lambda \mu} E_{1}(\lambda) E_{2}(\mu)=\left(E_{1}(\lambda+\Delta \lambda)-E_{1}(\lambda)\right)\left(E_{2}(\mu+\Delta \mu)-E_{2}(\mu)\right)$. In a sense the normal transformation is the plane analogue of the Hermitian.

In particular if $T$ is unitary $U$, then $I-\lambda U$ has a reciprocal for all values of $\lambda$ not on the unit circle in the complex plane. Utilizing this fact, and performing a transformation to polar coordinates, we see that to every unitary transformation there corresponds a spectral set $E(\lambda)$ on the interval $(0,2 \pi)$ such that

$$
U \xi=\int_{0}^{2 \pi} e^{i \lambda} d_{\lambda} E(\lambda) \xi
$$

and conversely. This suggests that the unitary transformations are connected with the Hermitian by a transformation of the symbolic form $U=e^{i H}$. $\dagger$

Obviously this method of representation can be extended to multiplicative combinations of Hermitian, unitary, and normal transformations.

9. Unlimited Transformations. After Hilbert the first steps in the discussion of such transformations were taken by Carleman. The introduction of infinite matrices as a tool in atomic theory gave a new impulse towards the investigation of such transformations. So far the chief contributors have been von Neumann, Stone, and Wintner.

The transformations so far treated have been limited and de-

$\dagger$ See J. von Neumann, loc. cit., pp. 119, etc.; Wintner, Mathematische Zeitschrift, pp. 268, etc. 
fined for the entire space. If we drop the second of these conditions, then it seems natural to demand at least that a transformation be defined and linear on a linear subset $\mathfrak{F}$ dense in $\mathfrak{S}$. (For example, $i d / d x$ on the class of polynomials, dense in continuous function set.) If in addition the transformation $T$ is limited on a set $\mathfrak{F}$ dense in $\mathfrak{S}$, it is uniformly continuous on this set and so can be extended to a continuous, that is, a limited transformation on $\mathfrak{S}$. Consequently if a transformation is not limited, there exists a subset of $\mathfrak{S}$ dense in $\mathfrak{S}$, on which it is not limited.

To define the adjoint of a transformation $T$, we select the elements $\eta$ of $\mathfrak{S}$ for which $(T \xi, \eta)$ is a linear limited operation on $\mathfrak{F}$, so that there exists an element $\eta^{*}$ such that $(T \xi, \eta)$ $=\left(\xi, \eta^{*}\right)$ for all $\xi$ of $\mathfrak{F}$. We thus define $T^{*} \eta=\eta^{*}$ on $\mathfrak{F}^{*}$. If $\mathfrak{F}^{*}$ is dense on $\mathfrak{S}$, then we can similarly define $T^{* *}$ and $\mathfrak{F}^{* *}$, and $\mathfrak{F}^{* *}$ will include $\mathfrak{F}$. If in particular $\mathfrak{F}^{*}=\mathfrak{F}$ and $T^{*}=T$, then $T$ is said to be Hermitian (von Neumann: hypermaximal; Stone: self-adjoint) and denoted by $H$. A Hermitian transformation is said to be commutative with a limited transformation $T$ if both $\xi$ and $T \xi$ belong to $\mathfrak{F}$ and $H T \xi=T H \xi$ on $\mathfrak{F}$.

As in the case of limited transformations, if $H$ is Hermitian on $\mathfrak{F}$, then for all $\lambda$ which are not real the transformation $I-\lambda H$ possesses a reciprocal which is a limited transformation on $\mathfrak{S}$. In particular $I+i H$ and $I-i H$ have reciprocals $B$ and $B^{*}$, which are adjoint. Riesz $\dagger$ observes that if one defines $E_{-}$and $E_{+}$for the transformation

$$
D=B+B^{*}=2 B^{*} H B,
$$

then these will serve also as the $E_{-}$and $E_{+}$for the transformation $H$, so that it is possible to define the transformations $E(\lambda)$ for the transformation $I-\lambda H, \lambda$ being real. The final result is as follows.

THEOREM 4. Every Hermitian transformation gives rise to a spectral set $E(\lambda)$, defined on $(-\infty, \infty)$, zero for $\lambda=-\infty$ and the identity for $\lambda=\infty$, such that a necessary and sufficient condition that $\xi$ belong to $\mathfrak{F}$, that is, $H$ be defined for $\xi$, is that the Stieltjes integral

$$
\int_{-\infty}^{\infty}|\lambda|{ }^{2} d_{\lambda}(E(\lambda), \xi)
$$

† Szeged Acta, p. 44. 
exist, the integral being defined as

$$
\lim _{\max \Delta_{i} \lambda \rightarrow 0} \sum_{i=-\infty}^{\infty}\left|l_{i}\right|^{2} \Delta_{i}(E(\lambda) \xi, \xi) .
$$

Then $H \xi=\int_{-\infty}^{\infty} \lambda d_{\lambda} E(\lambda) \xi$, existent in the sense of norms, and

$$
(I-\mu H)^{-1}=\int_{-\infty}^{\infty} \frac{d_{\lambda} E(\lambda)}{\lambda-\mu}
$$

where it exists.

J. von Neumann $\dagger$ obtains the same result another way. Essentially, his method involves the observation that $I+i H$ and $I-i H$ having limited reciprocals, each transforms $\mathfrak{F}$ into $\mathfrak{S}$, and therefore they define a limited transformation $U$ which is unitary. Solving the symbolic equation

$$
U(I+i H)=I-i H,
$$

for $H$ and applying the result to the canonical representation of a $U$, namely, $\int_{0}^{2 \pi} e^{i \lambda} d E(\lambda)$ or $\int_{-\pi}^{\pi} e^{i \lambda} d E(\lambda)$, suggests the transformation $\lambda=2$ arctan $\rho$, which leads to the same final characterization of the field $\mathfrak{F}$ and the transformation $H$. Stone $\ddagger$ obtains the same result by working through a representation for the reciprocal of $I-\lambda H$ for $\lambda$ not real.

It is perhaps interesting to note that the sums involved in defining the set $\mathfrak{F}$ of elements $\xi$ for which $H$ is defined are analogous to the one involved in defining the set of Lebesgue integrable square functions, the value $(E(\lambda) \xi, \xi)$ replacing the measure of the set $E$ for which $f \leqq \lambda$; further that the set $\mathfrak{F}$ of elements $\xi$ form again a Hilbert space, the Hermitian operator being

$$
[\xi, \eta]=\int_{-\infty}^{\infty}|\lambda|^{2} d_{\lambda}(E(\lambda) \xi, E(\lambda) \eta),
$$

but the elements $H \xi$ do not necessarily belong to this space.

J. von Neumann has investigated further transformations which satisfy the Hermitian condition for only a part of the set on which they are definable. For these the set $\mathfrak{F}^{*}$ would con-

$\dagger$ Loc. cit., pp. 80, 92.

$\ddagger$ Loc. cit., pp. $423-425$. 
tain $\mathfrak{F}$, and on $\mathfrak{F}$ we have $T \xi=T^{*} \xi$, but the transformation $T^{*}$ is not Hermitian, that is, there exists an $\eta$ of $\mathfrak{S}$ for which $\left(\eta, T^{*} \eta\right)$ is not real. If $\mathfrak{F}$ is the set on which $\left(\eta, T^{*} \eta\right)$ is real, then $T$ is said to be maximal. He finds by utilizing the correspondence defined by $I+i T$ and $I-i T$, called the Cayley transform, that the maximal transformations are expressible as the sum of a Hermitian transformation and a finite or denumerable set of transformations for which the Cayley transformation has for a certain normed orthogonal system of elements $\phi_{1}, \cdots, \phi_{n}, \cdots$ the form

$$
U \phi_{n}=\phi_{n-1} .
$$

J. von Neumann has considered further the transformations which as given are neither Hermitian nor maximal, and finds very interesting properties, particularly with respect to a certain amount of indefiniteness in the singularities of their reciprocals, first observed by Carleman.

It is to be hoped that the impetus given by these recent researches will be effective in encouraging further investigation into the characteristics of general limited and unlimited transformations in $\mathfrak{S}$ spaces and general vector spaces.

The University of Michigan 